This item was submitted to Loughborough's Research Repository by the author.

Items in Figshare are protected by copyright, with all rights reserved, unless otherwise indicated.

\title{
Dispersion and microwave processing of nano-sized ITO powder for the fabrication of transparent conductive oxides
}

PLEASE CITE THE PUBLISHED VERSION

http://dx.doi.org/10.1016/j.ceramint.2016.08.157

PUBLISHER

(C) Elsevier

VERSION

AM (Accepted Manuscript)

\section{PUBLISHER STATEMENT}

This work is made available according to the conditions of the Creative Commons Attribution-NonCommercialNoDerivatives 4.0 International (CC BY-NC-ND 4.0) licence. Full details of this licence are available at: https://creativecommons.org/licenses/by-nc-nd/4.0/

\section{LICENCE}

CC BY-NC-ND 4.0

\section{REPOSITORY RECORD}

Ghanizadeh, Shaghayegh, Nirmal Peiris, D.S.Y. Jayathilake, David Hutt, Upul Wijayantha-Kahagala-Gamage, Darren Southee, Paul Conway, et al.. 2019. "Dispersion and Microwave Processing of Nano-sized ITO Powder for the Fabrication of Transparent Conductive Oxides". figshare. https://hdl.handle.net/2134/22724. 


\title{
Dispersion and Microwave Processing of Nano-sized ITO Powder for the Fabrication of Transparent Conductive Oxides
}

\author{
S. Ghanizadeh ${ }^{1^{*}}$, T.A. Nirmal Peiris ${ }^{2}$, D.S.Y. Jayathilake ${ }^{2}$, D.A. Hutt ${ }^{1}$, K.G.U. Wijayantha ${ }^{2}$, D.J. Southee ${ }^{3}$, P.P. \\ Conway $^{1}$, P. Marchand ${ }^{4}$, J.A. Darr ${ }^{4}$, I.P. Parkin ${ }^{4}$, C.J. Carmalt ${ }^{4}$ \\ ${ }^{1}$ Wolfson School of Mechanical, Electrical and Manufacturing Engineering, Loughborough University, \\ Loughborough LE11 3TU, UK \\ 2 Energy Research Laboratory, Department of Chemistry, Loughborough University, Loughborough LE11 $3 T$, \\ UK \\ ${ }^{3}$ Design School, Loughborough University, Loughborough LE11 3TU, UK \\ ${ }^{4}$ Department of Chemistry, University College London, London WC1H OAJ, UK \\ * Corresponding author: Shaghayegh Ghanizadeh, S.Ghanizadeh@lboro.ac.uk
}

\begin{abstract}
Aqueous dispersions of tin-doped indium oxide (ITO) nanopowder were prepared and the effect of the addition of PEG 400, Tween 80 and $\beta$-alanine as dispersants was investigated using zeta potential and particle size distribution measurements. Both PEG 400 and $\beta$-alanine were found to produce stable dispersions that were used to deposit ITO thin films on glass substrates by dip and spin coating methods. The ITO thin films were heat-treated using both conventional and microwave heat treatment in order to improve the inter-particle connections and hence the resistivity and transparency of the films. All the films exhibited an average transmittance of $>80 \%$ over the visible spectrum after being subjected to the heat treatment process. ITO films prepared with no dispersant showed very high resistivity values for both heating methods, however addition of 2 wt\% PEG 400 to the dispersion yielded a reduction in the resistivity values to $1.4 \times 10^{-1} \Omega \mathrm{cm}$ and $3.8 \times 10^{-2} \Omega \mathrm{cm}$ for conventionally and microwave treated films, respectively. The surface morphological studies confirmed that addition of dispersants improved the film uniformity and inter-particle connections of the ITO films considerably.
\end{abstract}

Keywords: Nanoparticles; Thin films; Indium/Indium compounds; Transparent conducting oxides; Dispersants/Dispersion 


\section{Introduction}

Tin-doped indium oxide (ITO; $\ln _{2} \mathrm{O}_{3}: \mathrm{Sn}$ ) thin films have attracted considerable attention for many years due to their characteristics of high optical transparency in the visible region, reflectance in the IR range and good electrical conductivity [1-4]. This combination of electrical and optical properties enables these thin films to be used in a range of applications including touch screens and flat panel displays, electrochromic displays (ECDs) and liquid crystal displays (LCDs), as well as in energy-saving glazing, solar cells and gas sensors [35].

ITO thin film patterns are prepared conventionally by vacuum processes such as sputtering or evaporation often followed by photolithography and chemical etching [6]. However these methods have disadvantages, for example, they can be wasteful of materials due to ITO deposition onto unwanted areas and vacuum processes require significant infrastructure and can be slow. The limited availability of indium is also seen as a serious global issue, which is being exacerbated by the rapid growth of the many applications, resulting in significant increases in the cost of indium. A reduction of indium consumption is therefore an urgent requirement that could be achieved, for instance, by direct deposition and patterning of ITO on surfaces, avoiding the loss of material typically encountered in vacuum deposition processes. One approach to this is through the use of ITO nanoparticle inks or dispersions that may be screen or inkjet printed [7]. For example, ITO coatings have been prepared using direct gravure printing on polymer substrates such as polyethylene naphthalate (PEN) $[8,9]$, where, in some studies, the concentration of the ITO dispersions was greater than 50 wt\% [9]. Spin and dip coating, two of the simplest deposition techniques available [10], have also been used to directly deposit ITO particles dispersed in water and organic vehicles, such as ethanol, on glass substrates [1,5] and can lead to an economical process for the production of ITO films. However, in all these methods, the level of agglomeration existing in the powders used to prepare these inks/pastes can affect the quality of the coatings produced. 
After deposition, heat-treatment of the printed ITO nanoparticle thin films is necessary to bring the particles into contact and ensure high mobility of charge carriers through the film, thereby lowering the electrical resistivity. Published methods include conventional heating and microwave processing of the films in air or a reducing atmosphere environment $[1,7,11,12]$. Post-treatment of the films under a reducing atmosphere has been shown to enhance the carrier density and decrease the resistivity as a result [1]. Furthermore, the temperature of the heating process can be lowered by reducing the dimensions of the particulate material from microscale to nanoscale; this leads to a considerably higher fraction of grain boundaries and causes many short-circuit diffusion paths during densification [13].

Irradiation with microwaves as an alternative heat-treatment technique has received a great deal of interest and has been reported to improve the particle necking and crystallinity with minimal thermal stress in the substrate $[14,15]$. This is due to the generation of an entirely different heating profile by microwave irradiation in comparison with that of conventional heating. Microwaves interact with lossy materials (materials with medium-range electrical conductivity) and can generate rapid and uniform heating throughout the material, which leads to minimisation of the thermal gradient and the processing time $[16,17]$. In addition, it has been reported that some ceramic materials, such as $\mathrm{TiO}_{2}$, synthesised using low temperature methods can be partially amorphous; this can be solved by microwave irradiation of the film without affecting its micro- or nano-sized structure [15].

In the work reported here, ITO thin films have been prepared by direct deposition methods including dip and spin coating of nanoparticle ITO inks with solid content values of $15 \mathrm{wt} \%$. Other published studies have used nanoparticles prepared by sol-gel or precipitation methods that can lead to a high level of agglomeration due to the drying and calcination stages. Therefore this work used nanopowders of ITO (with 10 at\% Sn) that were directly synthesised as nanoparticles dispersed in a liquid media, using a continuous hydrothermal flow synthesis (CHFS) method, as discussed in previous work $[4,18,19]$. The effect of the addition of different dispersants on the level of agglomeration of the nanopowders along with 
a comparative study of conventional and microwave heating techniques on the characteristics of the films was investigated.

\section{Experimental Procedure}

The CHFS ITO nanopowder [4] was dispersed in deionised (DI) water with additions of different dispersants including PEG 400 (Polyethylene glycol, 95+\%, Sigma-Aldrich, UK), $\beta$ alanine ( $\beta$ amino acid, 98\%, Sigma-Aldrich, UK) and Tween 80 (Sigma-Aldrich, UK). First, appropriate quantities of DI water and each dispersant were mixed and stirred for $1 \mathrm{~h}$ using a magnetic stirrer. The amount of dispersant used was either 1 or 2 wt $\%$ relative to the amount of ITO nanopowder. The ITO nanopowder was then added and the mixture was stirred for a further 2 hours in order to achieve a homogeneous suspension. The suspensions were then subjected to ultrasonic agitation using a SoniPrep ultrasound unit (MSE Ltd, London, UK) using $150 \mathrm{~W}$ at $15 \mathrm{kHz}$ for $30 \mathrm{~s}$, after which the beakers were sealed to prevent liquid evaporation and then left for $1 \mathrm{~h}$ to equilibrate.

The prepared dispersions were characterised for zeta potential and particle size distribution using a Zetamaster system (Malvern Instruments, Malvern, UK). In order to do this, a sample of dispersion was taken and diluted with DI water to $\leq 0.1$ wt\%. Rheological behaviour analysis of the suspensions was determined using an Anton Paar RheolabQC cup and cone rotational rheometer (Anton Paar, Austria). Thermogravimetric analysis (TGA) was carried out on a quantity of the dispersion that was dried at $100^{\circ} \mathrm{C}$ for over $12 \mathrm{hr}$. A SDT 2960 Simultaneous TGA/DSC (TA instruments, U.K Ltd.) was used for the measurements with the sample heated up to $800^{\circ} \mathrm{C}$ in air with a ramp rate of $10^{\circ} \mathrm{C} \mathrm{min}^{-1}$.

ITO thin films were fabricated using the prepared suspensions via both dip and spin coating techniques on glass substrates (NSG, Pilkington, UK) of $20 \times 10 \mathrm{~mm}^{2}$, which were cleaned thoroughly using DI water, acetone, isopropanol and ethanol prior to the deposition process. Using the prepared dispersions, ITO films were dip-coated by immersing the glass substrates vertically into the dispersion for $10 \mathrm{~s}$, followed by withdrawing and placing them 
horizontally on a flat surface to dry at room temperature. The spin coating process was carried out using a KW4A spin coater (Chemat Technology, Northridge, US) by dropping ITO dispersions (approx. 1 drop s$^{-1}$ ) onto the spinning slides in 3 or 6 cycles of $10 \mathrm{~s}$ with a spin speed of $2000 \mathrm{rpm}$. After spin coating, the deposited ITO films were left to dry on a flat surface for $\sim 24 \mathrm{~h}$ at room temperature.

Conventional heating was carried out in a tube furnace (MTF-10-25-130, Carbolite, UK) with a ramp rate of $15^{\circ} \mathrm{C} \min ^{-1}$ to $600^{\circ} \mathrm{C}$ followed by a dwell time of $3 \mathrm{~h}$. During this process, a flow of $\mathrm{N}_{2}$ (purity $>99 \%, \mathrm{BOC}$ ) was passed through the furnace at a rate of $1825 \mathrm{~mL} \mathrm{~min}{ }^{-1}$. After heat treatment, the furnace was turned off and allowed to cool to room temperature under a continual $\mathrm{N}_{2}$ flow. Microwave-assisted heat-treatment was performed using a microwave oven (Microwave Research Applications Inc. BP-211/50, USA) operating at a frequency of $2.45 \mathrm{GHz}$ with a maximum power of $3000 \mathrm{~W}$. Heating of the ITO films was assisted by using SiC susceptor tiles placed on the top and bottom of the coated substrates and the heat loss during annealing was minimized by surrounding the sample with insulator blocks. The microwave treatment of the films was carried out under a similar atmosphere to the conventional process with microwave power applied for a period of $90 \mathrm{~s}$, which raised the temperature of the sample from room temperature to $600^{\circ} \mathrm{C}$, at which point the sample was allowed to cool to room temperature over a period of $\sim 15 \mathrm{~min}$. The temperature of the SiC susceptor was monitored during this process using an infrared temperature probe (Mikron infrared Inc. M67/M67S series, USA) with an error of $\pm 5^{\circ} \mathrm{C}$.

FEG-SEM (Leo 1530VP FEGSEM, LEO Elektronenskopie GmbH, Oberkochen, Germany) was used to examine the surface morphology of the films. Cross-sections were prepared either by scribing and breaking the glass substrate, or by milling using a focussed ion beam, FIB-SEM system (Nova200 NanoLab, FEI, US). For FIB-SEM the specimen was tilted $52^{\circ}$ with respect to the SEM optics for imaging and therefore the resultant SEM micrographs were compressed along the $Y$ axis so that all the dimensions obtained from them were 
corrected using the equation: $Y_{c}=Y_{r} / \sin 52^{\circ}$, where $Y_{r}$ is the obtained dimension from the micrograph and $Y_{c}$ is the corrected value. The phase and crystallinity of the films were studied using a Bruker D8 XRD (Bruker AXS GmbH, Karlsruhe, Germany), operating with monochromatic $\mathrm{Cu} \mathrm{Ka}(\lambda=1.54 \AA)$ radiation and a position sensitive detector. X-ray photoelectron spectroscopy (XPS) was performed using a Thermo Scientific Ka photoelectron spectrometer (Thermo Fisher Scientific, UK) using monochromatic Al Ka radiation. The transmittance of the films was determined using a Lambda 35 spectrophotometer (Perkin Elmer, Massachusetts, US); here the transmittance of the glass substrate was measured initially and used as a reference. The sheet resistance $\left(R_{s}\right)$ was measured using a Jandel (HM20) 4-point probe test unit (Jandel Engineering Ltd., Linslade, UK) and the resistivity was calculated according to the equation $\rho=R_{s} t$, where $\rho$ is the resistivity, $R_{\mathrm{S}}$ is the measured sheet resistance and $t$ is the thickness of the film.

\section{Results and Discussion}

\subsection{Dispersion Preparation and Characterisation}

The ITO nanopowder (10 at\% Sn) prepared by continuous hydrothermal flow synthesis [4] was initially pale blue in colour with a mean particle size of $\sim 15 \mathrm{~nm}$ and mixed morphology of spherical and rounded cube-like particles. The zeta potential of the ITO nanoparticles in the dispersions was measured and is presented in Table 1. For all of the dispersions prepared, the ITO particles acquired a positive charge of over $35 \mathrm{mV}$; these values are similar to those published in the study by Reindl, et al. [20], where the zeta potential of an ITO dispersion at a $\mathrm{pH}$ of $\sim 5.7$ was found to be $\sim 25 \mathrm{mV}$. The addition of non-ionic and Zwitterion dispersants did not affect the magnitude of the zeta potential of the ITO dispersions and this value was approximately constant $(\sim 40 \mathrm{mV})$ for all of the dispersions. However the addition of $\beta$-alanine yielded an almost neutral $\mathrm{pH}$ value compared to those with non-ionic dispersants or no dispersant; this could be due to the amphoteric nature of $\beta$-alanine. According to the Deryaguin-Landau-Verwey-Overbeek (DLVO) theory the relationship between van der Waals attractive and electrostatic repulsive forces determines the stability of aqueous 
dispersions of nano and submicron-sized particles [21,22]. If the van der Waals attraction is less than the electrostatic repulsion, the dispersion is considered as stable and to form agglomerates, the particles have to pass the energy barrier of electrostatic repulsion in order to come close and join each other. In the dispersions prepared here, the magnitude of the zeta potential is significant and would be expected to lead to stable dispersions [21].

The particle size distributions of the ITO suspensions both with and without $2 \mathrm{wt} \%$ of different dispersants are presented in Figure 1 in volume\%. It can be seen that bimodal distributions were obtained in each case, but the level of large agglomerates and smallest particle sizes varied with the dispersant. In the absence of dispersants, the high surface energy of the nanoparticles resulted in a tendency to agglomerate. Adding $1 \mathrm{wt} \%$ of each of the dispersants did not improve the size distribution of the ITO nanoparticles, while increasing the amount of dispersant to $2 \mathrm{wt} \%$ resulted in a lower level of particle agglomeration. $2 \mathrm{wt} \%$ of dispersant was therefore used for the subsequent particle deposition trials presented here. In particular, the use of $\beta$-alanine shifted the mean peak the most towards smaller particle sizes, as demonstrated in Figure 1. However, there is still a considerable difference between the value these peaks are assigned to and the primary particle size observed in TEM micrographs published in previous work [4]. The lower particle size obtained with $\beta$-alanine may be partly due to its lower molecular weight of $\sim 89 \mathrm{~g} \mathrm{~mol}^{-1}$ compared to the other two dispersants.

Figure 2-a shows the rheological behaviour (increasing and decreasing shear rate curves) of viscosity vs shear rate, for the dispersions prepared with PEG and $\beta$-alanine, which were previously shown to have lower levels of particle agglomeration: the viscosity of the aqueous dispersions did not change noticeably as dispersants were introduced. Figure 2-b presents the shear stress vs shear rate plot for the $15 \mathrm{wt} \%$ ITO suspensions. All the dispersions displayed near-Newtonian flow behaviour over the range of shear rates investigated. The rheological behaviour and viscosity values of $\sim 3.5 \mathrm{mPa} s$ (for a shear rate of $150 \mathrm{~s}^{-1}$ ) indicate that all the dispersions have the potential to form uniform films by spin coating at a 
speed of $2000 \mathrm{rpm}$, as demonstrated by Huang and Chou [23]. Their study on the spin coating process of silica films showed that for a spin speed of $2000 \mathrm{rpm}$, a process window in terms of viscosity in the range of $\sim 3.3-5.7 \mathrm{mPa} s$ can be defined to achieve uniform and defect free films. They also showed that it was challenging to deposit films of good quality using dispersions with values of viscosity that were either too low or too high.

\subsection{Fabrication of ITO Thin Films}

ITO thin films were deposited on glass substrates from dispersions of $15 \mathrm{wt} \%$ solids content using the dip coating method and resulted in ITO nanoparticle films with an average thickness of $1-2.3 \mu \mathrm{m}$ after drying, as shown in Figure 3. A high level of agglomeration and roughness was observed for the film deposited using the suspension with no added dispersant (thickness of $\sim 1 \mu \mathrm{m}$ excluding the agglomerates present on the surface), which is in line with the particle size distribution that showed many large agglomerates. In contrast, the use of $\beta$-alanine as a dispersant resulted in a more uniform coating (thickness of $\sim 1.1$ $\mu \mathrm{m})$, although the removal of flakes of the material from the edge of the cross-section (Figure 3-b) indicate that there was poor adherence between the film and the glass substrate. Dip coating using the PEG dispersion resulted in a continuous coating with the film still adhered to the substrate after cutting the samples and a uniform microstructure on the top surface, as seen in Figures 3-c and d.

Figure 4 shows the surface morphology of thin films obtained by spin coating of aqueous ITO suspensions with and without dispersant. As can be observed, using no dispersant led to a very rough surface, whilst the addition of $2 \mathrm{wt} \%$ of both PEG and $\beta$-alanine resulted in smoother films, in agreement with the dip coating observations and the particle size distributions. Use of 2 wt\% PEG also led to a better film coverage and higher compactness, similar to that observed for the dip-coated sample produced using the same dispersion. 


\subsection{Post-processing of Thin Films}

Post-treatment of the dip-coated samples in both microwave and conventional furnaces resulted in ITO films that exhibited cracking, which could be due to non-uniformity of the film thickness of the coatings. In the future, a potential solution to this may be to control the rate of drying of the coatings after the deposition process. SEM micrographs of the top surface and cross section (using FIB) of spin-coated films prepared using suspensions with 2 wt\% PEG then heat-treated by microwave processing and conventional heating are shown in Figures 5 and 6 . The micrographs indicate that the structure of the microwave treated film (with average corrected thickness value of $\sim 400 \mathrm{~nm}$ ) is more homogeneous compared to the conventionally treated one (with average corrected thickness value of $\sim 380 \mathrm{~nm}$ ); this can be due to the effect of the microwave irradiation on the improvement of the particle necking, while retaining the nanostructure. The cross-section micrographs demonstrate porosity in both samples and it did not appear to change with the introduction of microwave processing.

X-ray diffraction was used to determine the crystalline structure of films using $\ln _{2} \mathrm{O}_{3}$ and InOOH JCPDS data (JCPDS card no. 01-071-2194 and no. 01-071-2283 for $\ln _{2} \mathrm{O}_{3}$ and $\mathrm{InOOH}$, respectively) for comparison and to investigate how the heat-treatment affected the structure of the ITO. Figure 7 shows XRD data of the ITO films prepared by spin coating using the aqueous dispersion with 2 wt\% PEG, heat-treated via both microwave and conventional methods. The figure also shows data obtained from an as-deposited film before heat-treatment. Heat-treatment of the deposited films to $600^{\circ} \mathrm{C}$ under $N_{2}$ atmosphere developed the oxide phase in the post-treated samples with both microwave and conventional processes and resulted in films of pure indium tin oxide without traces of hydroxide phase. The elemental composition of heat treated films was also investigated by XPS, which suggested a Sn loading of 9.7 at\% and 10.1 at $\%$ for conventionally and microwave processed samples, respectively. The obtained values were close to the Sn content of the starting nanopowders of 10 at\% [4]; this confirmed that there was no evidence of dopant segregation to the surface of the films after being heat treated at $600^{\circ} \mathrm{C}$. 
Thermogravimetric analysis of pre-dried ITO dispersions with and without 2 wt $\%$ PEG 400 was carried out. The weight and derivative weight curves of these two samples in the temperature range of $50-800^{\circ} \mathrm{C}$ are displayed in Figure 8 . The sample with PEG showed two discrete decomposition steps up to $450^{\circ} \mathrm{C}$ with an overall weight loss of $\sim 12 \mathrm{wt} \%$. The first decomposition step took place over a short temperature range and was assigned to the removal of the physical and more strongly chemisorbed water and PEG present in the dried powder. The second, more gradual reduction in weight from 250 to $400^{\circ} \mathrm{C}$ followed a similar gradient as the sample without dispersant and indicates a change associated with the ITO itself. This is thought to be due to the thermal decomposition of the remaining $\mathrm{OH}$ groups of indium and tin hydroxides at high temperatures and their transformation to metal oxides, as reported in the literature [24] and in agreement with the changes observed in the XRD data (Figure 7).

\subsection{Optical and Electrical Characterisation of Thin Films}

Optical transmission spectra of ITO thin films spin coated (deposited for $6 \times 10 \mathrm{~s}$ ) on glass substrates and heat-treated are shown in Figure 9. All the films showed no sign of the previous blue colour of the original nanopowder and gave high transmittance, with average transmittance of $>85 \%$ over the wavelength range of 400 to $700 \mathrm{~nm}$. There was an absorption edge at $\sim 380 \mathrm{~nm}$, which is caused by electron excitation from a valence band to a conduction band. This transmittance data is in agreement with the reported values in previous studies $[5,12,25]$. Electrical conductivity measurement of the films prepared by spin coating (deposited for $6 \times 10 \mathrm{~s}$ ) using the dispersions with no added dispersant and $2 \mathrm{wt} \% \beta$ alanine displayed high values of sheet resistance of $\sim 2.5 \times 10^{3} \Omega \mathrm{sq}^{-1}$ and $\sim 1.6 \times 10^{3} \Omega \mathrm{sq}^{-1}$, respectively, after microwave post-treatment. These high values observed for the films deposited using dispersions with no added dispersant and with $\beta$-alanine can be due to the presence of agglomerated particles on the surface of the former sample and the existence of a high number of cracks on the latter. However, addition of 2 wt $\%$ of PEG 400 to the ITO 
dispersions resulted in a sheet resistance of $8.0 \times 10^{2} \Omega \mathrm{sq}^{-1}$ and $2.0 \times 10^{2} \Omega \mathrm{sq}^{-1}$ corresponding to resistivity values of $1.4 \times 10^{-1} \Omega \mathrm{cm}$ and $3.8 \times 10^{-2} \Omega \mathrm{cm}$ based on the mean film thickness of $\sim 1.75 \mu \mathrm{m}$ and $1.90 \mu \mathrm{m}$ for the conventionally and microwave treated films, respectively. The lower resistivity value of the microwave-treated sample can be due to the improved connections between particles as a result of the volumetric heating produced by the microwave effect. Kim, et al. [26] have reported resistivity values as low as $10^{-3} \Omega \mathrm{cm}$ for ITO films prepared using spin coating of nanoparticle-based dispersions, but post treated via radiant heating up to $950^{\circ} \mathrm{C}$ under a controlled atmosphere with oxygen partial pressure of $10^{-6}$ to $10^{2}$ Torr. In a parallel study using aerosol-assisted chemical transport (AACT) for the deposition of the same ITO nanopowders, optical transparency of over $85 \%$ and resistivity of $6 \times 10^{-2} \Omega \mathrm{cm}$ was obtained using the same microwave heating profile as described here [27].

\section{Conclusions}

Aqueous dispersions of ITO nanoparticles were prepared using a nano-sized ITO powder with and without dispersants. Characterisation of the nanodispersions revealed that the addition of the dispersants did not affect the zeta potential value significantly, however adding $2 \mathrm{wt} \%$ of $\beta$-alanine led to the most significant decrease in the mean agglomerate size. Near Newtonian behaviour was observed for the prepared aqueous dispersions both with and without added dispersant. Films of ITO were deposited by dip and spin-coating techniques and were further heat-treated to $600^{\circ} \mathrm{C}$ using conventional and microwave heating methods. The transmittance of the post-treated films was found to be over $80 \%$ in the visible wavelength range and $\mathrm{XRD}$ showed that the films were largely transformed to oxide after these heat-treatments. Electrical conductivity measurement of the films prepared from suspensions without added dispersant revealed a high value of sheet resistance for both conventionally and microwave treated films. By adding 2 wt $\%$ of PEG 400 to the ITO dispersions, a decrease in the resistivity to $3.8 \times 10^{-2} \Omega \mathrm{cm}$ with microwave processing was achieved, which was lower than the value of $1.4 \times 10^{-1} \Omega \mathrm{cm}$ obtained for a conventionally treated film. These resistivity values can be attributed to the amount of porosity present in 
the deposited films (as observed in FIB-SEM micrographs), which can result in lower mobility of charge carriers. Microwave heating can offer an alternative route to the manufacture of TCO thin films in significantly shorter time duration and further work is underway to optimise this process.

\section{Acknowledgements}

This research was funded by the UK Engineering and Physical Sciences Research Council (EPSRC) under grant number EP/L017709/1. The authors gratefully acknowledge the support of the industrial collaborators, especially Malvern Instruments Ltd., Sun Chemical Ltd. and NSG, Pilkington. The assistance received from all members of the Energy Research Laboratory in the Department of Chemistry, Loughborough University and the Loughborough Materials Characterisation Centre (LMCC) particularly Mr. Scott Doak and Mrs. Patricia Cropper is also acknowledged. 


\section{References:}

1. N. Al-Dahoudi and M. A. Aegerter, 'Comparative study of transparent conductive $\ln _{2} \mathrm{O}_{3}$ : $\mathrm{Sn}$ (ITO) coatings made using a sol and a nanoparticle suspension,' Thin Solid Films, 502 [1] 193-197 (2006).

2. W. J. Tseng and F. Tzeng, 'Effect of ammonium polyacrylate on dispersion and rheology of aqueous ITO nanoparticle colloids,' Colloids Surf. Physicochem. Eng. Aspects, 276 [1] 3439 (2006).

3. D. Wu, J. Liu and Y. Wang, 'Enhancing indium tin oxide (ITO) thin film adhesiveness using the coupling agent silane,' Appl. Surf. Sci., 256 [9] 2934-2938 (2010).

4. P. Marchand, N. M. Makwana, C. J. Tighe, R. I. Gruar, I. P. Parkin, C. J. Carmalt and J. A. Darr, 'High-throughput synthesis, screening and scale-up of optimised conducting indium tin oxides.' ACS Combinatorial Science, 18 (2) 130 (2016).

5. K. Nishio, T. Sei and T. Tsuchiya, 'Preparation and electrical properties of ITO thin films by dip-coating process,' J. Mater. Sci., 31 [7] 1761-1766 (1996).

6. K. Nakashima and Y. Kumahara, 'Effect of tin oxide dispersion on nodule formation in ITO sputtering,' Vacuum, 66 [3] 221-226 (2002).

7. S. Hong, J. Kim, J. Lim, G. Choi and M. Isshiki, 'Characteristics of printed thin films using indium tin oxide (ITO) ink,' Materials transactions, 51 [10] 1905-1908 (2010).

8. S. Heusing, P. W. de Oliveira, E. Kraker, A. Haase, C. Palfinger and M. Veith, 'Wet chemical deposited ITO coatings on flexible substrates for organic photodiodes,' Thin Solid Films, 518 [4] 1164-1169 (2009).

9. J. Puetz and M. A. Aegerter, 'Direct gravure printing of indium tin oxide nanoparticle patterns on polymer foils,' Thin Solid Films, 516 [14] 4495-4501 (2008).

10. Y. Takahashi, S. Okada, R. B. H. Tahar, K. Nakano, T. Ban and Y. Ohya, 'Dip-coating of ITO films,' J. Non Cryst. Solids, 218 129-134 (1997).

11. M. Boehme and C. Charton, 'Properties of ITO on PET film in dependence on the coating conditions and thermal processing,' Surface and Coatings technology, 200 [1] 932935 (2005).

12. M. Okuya, N. Ito and K. Shiozaki, 'ITO thin films prepared by a microwave heating,' Thin Solid Films, 515 [24] 8656-8659 (2007).

13. S. Ghanizadeh, X. Bao, B. Vaidhyanathan and J. Binner, 'Synthesis of nano $\alpha$-alumina powders using hydrothermal and precipitation routes: A comparative study,' Ceram. Int., 40 [1] 1311-1319 (2013).

14. D. E. Clark, D. C. Folz and J. K. West, 'Processing materials with microwave energy,' Materials Science and Engineering: A, 287 [2] 153-158 (2000). 
15. A. A. Tahir, T. A. Peiris and K. G. Wijayantha, 'Enhancement of photoelectrochemical performance of AACVD-produced $\mathrm{TiO}_{2}$ electrodes by microwave irradiation while preserving the nanostructure,' Chemical Vapor Deposition, 18 [4-6] 107-111 (2012).

16. I. Bilecka and $M$. Niederberger, 'Microwave chemistry for inorganic nanomaterials synthesis,' Nanoscale, 2 [8] 1358-1374 (2010).

17. P. Kalyani, N. Kalaiselvi and N. G. Renganathan, 'Microwave-assisted synthesis of $\mathrm{LiNiO}_{2}$-a preliminary investigation,' J. Power Sources, 123 [1] 53-60 (2003).

18. M. Lübke, J. Shin, P. Marchand, P. Shearing, D. Brett, Z. Liu and J. A. Darr, 'Highly pseudocapacitive $\mathrm{Nb}$-doped $\mathrm{TiO}_{2}$ high power anodes for lithium-ion batteries,' Journal of Materials Chemistry A, 294 94-102 (2015).

19. P. Ramanujam, B. Vaidhyanathan, J. G. P. Binner, S. Ghanizadeh and C. Spacie, 'Solvothermal nanoYAG synthesis: Mechanism and particle growth kinetics,' The Journal of Supercritical Fluids, 107 433-440 (2016).

20. A. Reindl, M. Mahajeri, J. Hanft and W. Peukert, 'The influence of dispersing and stabilizing of indium tin oxide nanoparticles upon the characteristic properties of thin films,' Thin Solid Films, 517 [5] 1624-1629 (2009).

21. B. Derjaguin and L. Landau, 'Theory of the stability of strongly charged lyophobic sols and of the adhesion of strongly charged particles in solutions of electrolytes,' Prog Surf Sci, 43 [1-4] 30-59 (1993).

22. E. J. W. Verwey and J. T. G. Overbeek, 'Stability of lyophobic colloids,' (1948).

23. Y. Huang and K. Chou, 'Studies on the spin coating process of silica films,' Ceram. Int., 29 [5] 485-493 (2003).

24. N. C. Pramanik, S. Das and P. K. Biswas, 'The effect of Sn (IV) on transformation of coprecipitated hydrated In (III) and Sn (IV) hydroxides to indium tin oxide (ITO) powder,' Mater Lett, 56 [5] 671-679 (2002).

25. S. Hong, Y. Kim and J. Han, 'Development of ultrafine indium tin oxide (ITO) nanoparticle for ink-jet printing by low-temperature synthetic method,' Nanotechnology, IEEE Transactions on, 7 [2] 172-176 (2008).

26. N. Kim, J. Lee, Y. Lee, D. Nam, H. Yeon, S. Lee, T. Yang, Y. Lee, A. Chu and K. T. Nam, 'Enhanced conductivity of solution-processed indium tin oxide nanoparticle films by oxygen partial pressure controlled annealing,' Journal of Materials Chemistry C, 1 [37] 59535959 (2013).

27. T. A. N. Peiris, S. Ghanizadeh, D. S. Y. Jayathilake, D. A. Hutt, K. G. U. Wijayantha, Conway, P. P., Southee, D. J., I. P. Parkin, P. Marchand, J. A. Darr and C. J. Carmalt, 'Aerosol-assisted fabrication of tin-doped indium oxide ceramic thin films from nanoparticle suspensions,' J. Mater. Chem. C, [4] 5739-5746 (2016). 


\section{Tables:}

Table 1: Zeta potential ( $\pm 1 \mathrm{mV})$ and $\mathrm{pH}$ values of $15 \mathrm{wt} \%$ ITO suspensions.

\begin{tabular}{|c|c|c|}
\hline Suspension & Zeta potential I mV & $\mathrm{pH}$ \\
\hline No dispersant & 39 & 5.5 \\
\hline 1 wt\% PEG-400 & 40 & 5.6 \\
\hline 2 wt\% PEG-400 & 38 & 5.4 \\
\hline 1 wt\% Tween-80 & 39 & 5.5 \\
\hline 2 wt\% Tween-80 & 41 & 5.4 \\
\hline 1 wt\% $\beta$-alanine & 39 & 7.2 \\
\hline 2 wt\% $\beta$-alanine & 42 & 7.2 \\
\hline
\end{tabular}

Figures:

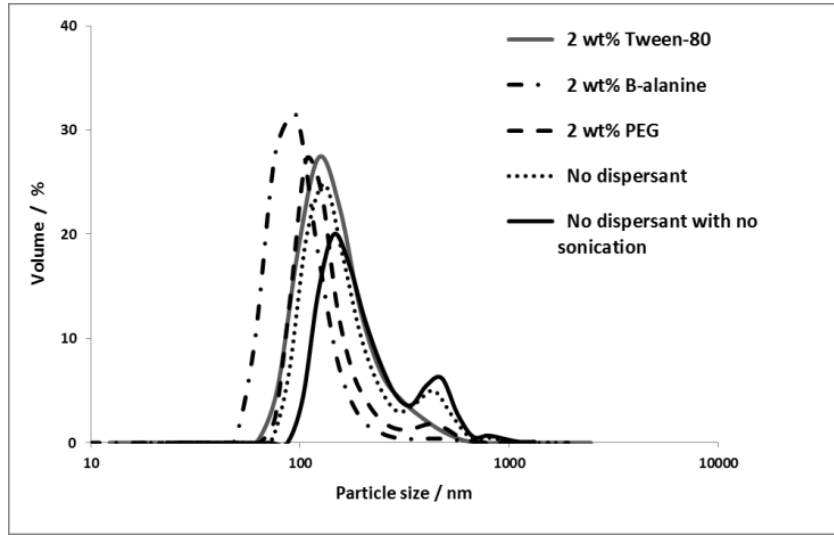

Figure 1: Particle size distribution of 15 wt $\%$ ITO dispersions.

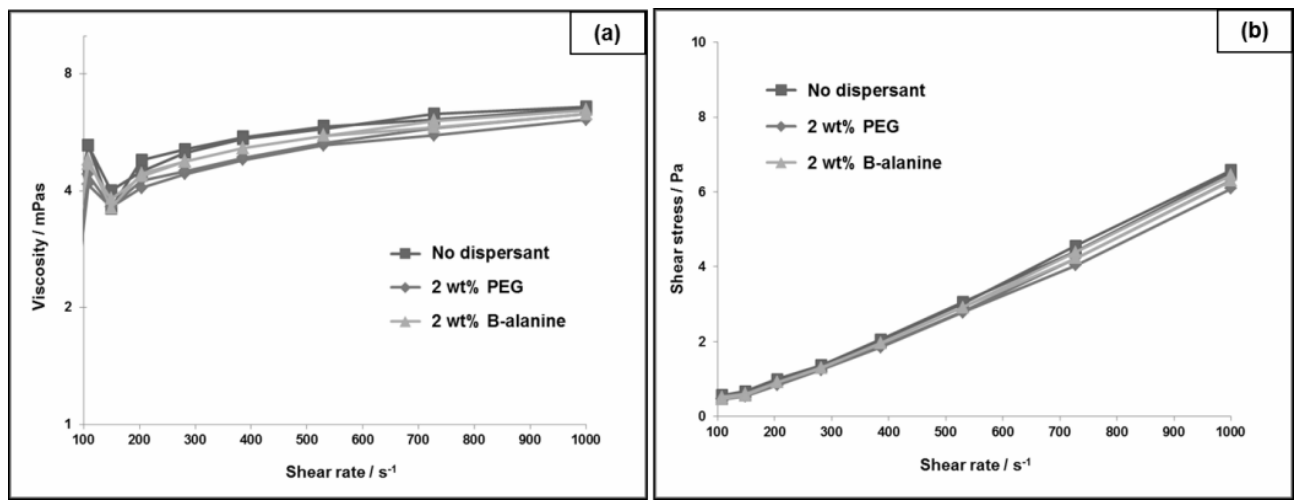

Figure 2: Rheological behaviour analysis of $15 \mathrm{wt} \%$ ITO dispersions: (a) viscosity vs. shear rate and (b) shear stress vs. shear rate. 

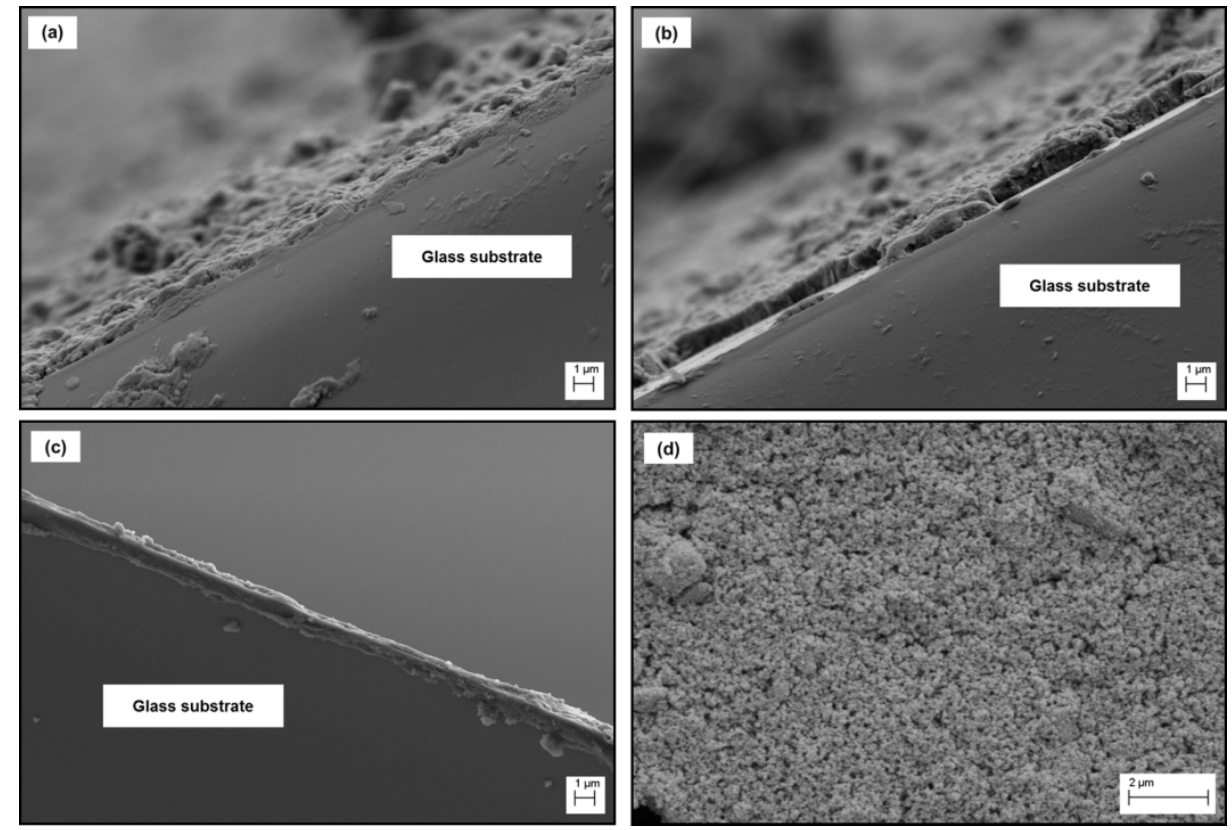

Figure 3: FEG-SEM micrographs of as-deposited samples prepared with the dip coating technique. Cross sections with (a) no dispersant, (b) $2 \mathrm{wt} \% \beta$-alanine, (c) $2 \mathrm{wt} \%$ PEG and (d) top surface view of the sample with $2 \mathrm{wt} \%$ PEG.

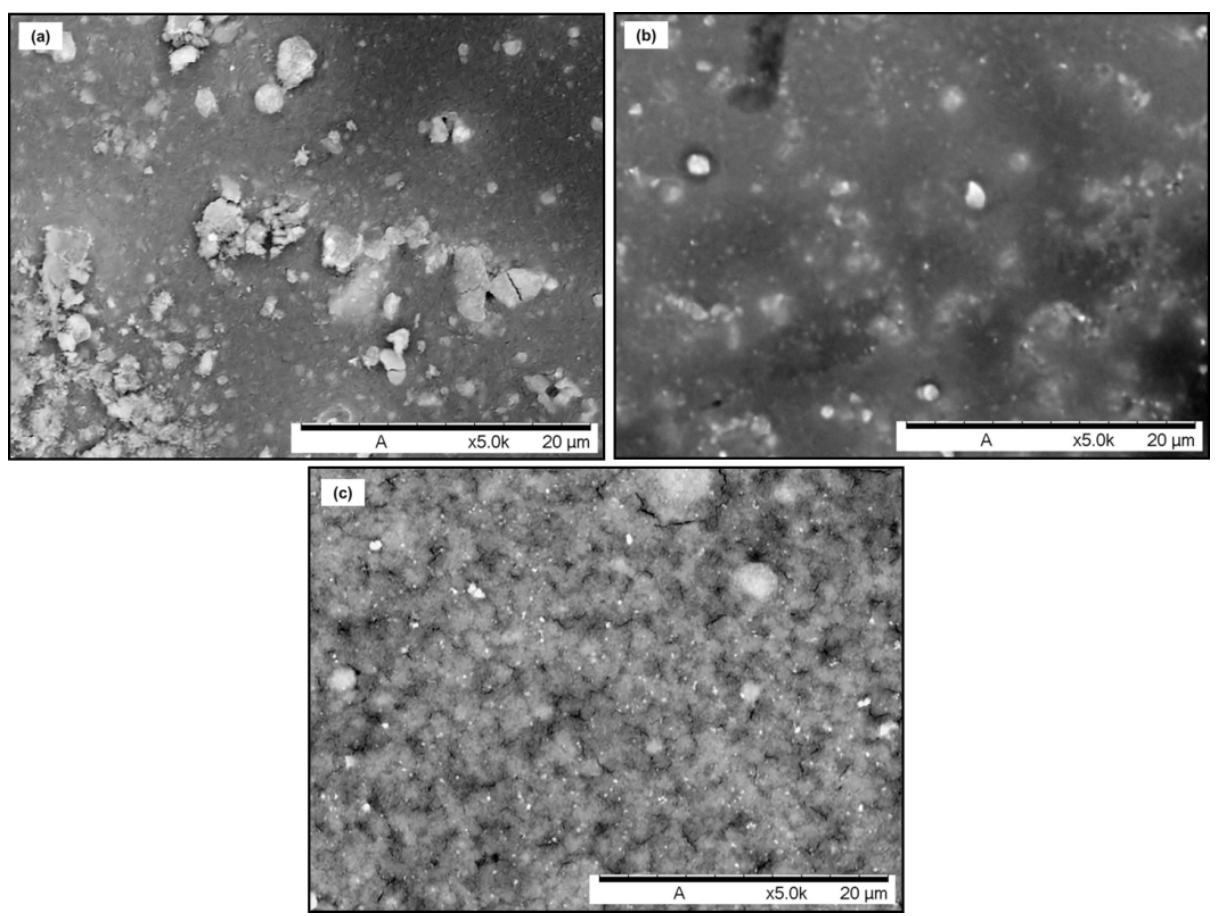

Figure 4: SEM micrographs of as-deposited films prepared via the spin coating technique (deposited for $6 \times 10$ s) with: (a) no dispersant, (b) 2 wt $\%$ PEG and (c) 2 wt $\% \beta$-alanine 


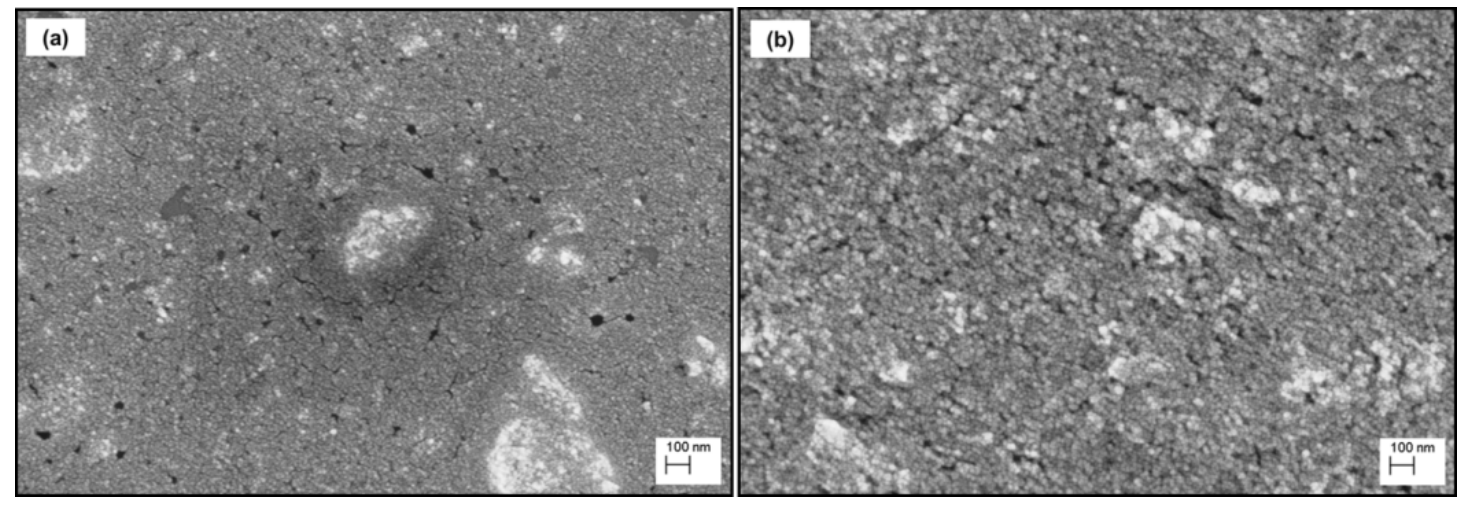

Figure 5: FEG-SEM micrographs of the top surface of spin-coated (deposited for $6 \times 10 \mathrm{~s}$ ) films prepared using suspensions with 2 wt\% PEG: (a) conventionally heat-treated and (b) microwave processed.
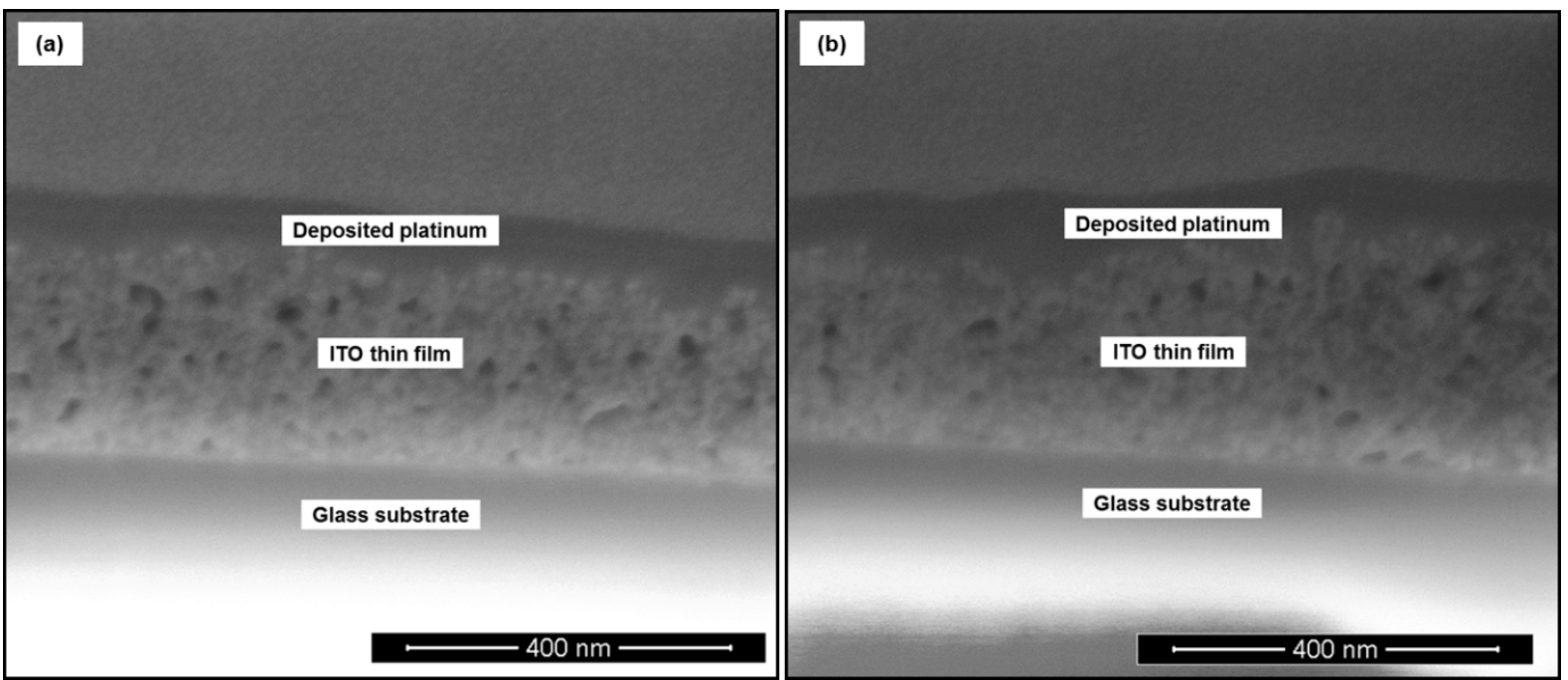

Figure 6: SEM micrographs of the cross section (prepared using FIB) of spin-coated (deposited for 3 $x$ 10s) films prepared using suspensions with 2 wt\% PEG: (a) conventionally heat-treated and (b) microwave processed. 


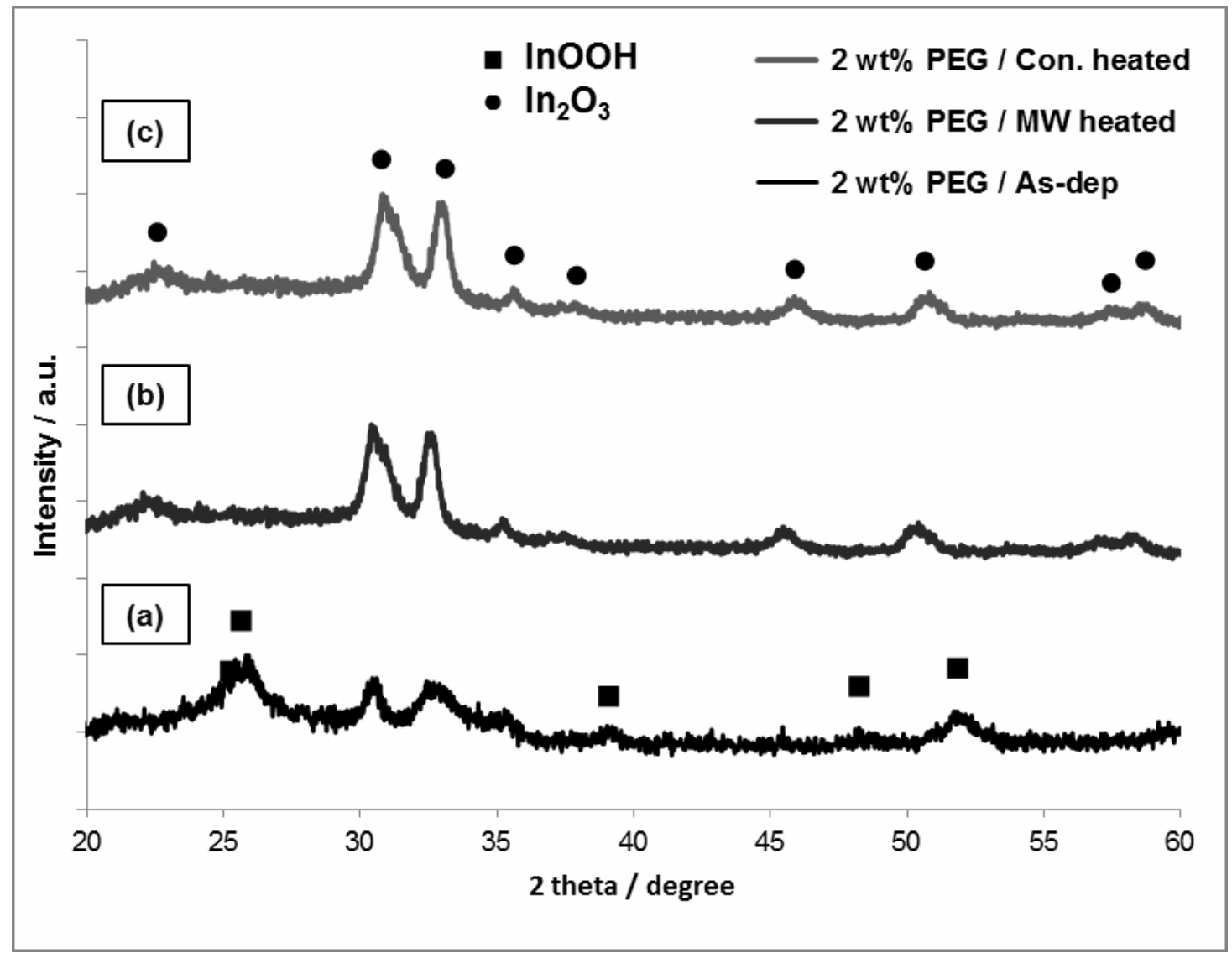

Figure 7: XRD patterns of as-deposited and heat treated ITO films: (a) as-deposited, (b) microwave processed and (c) conventionally heated for $3 \mathrm{~h}$.

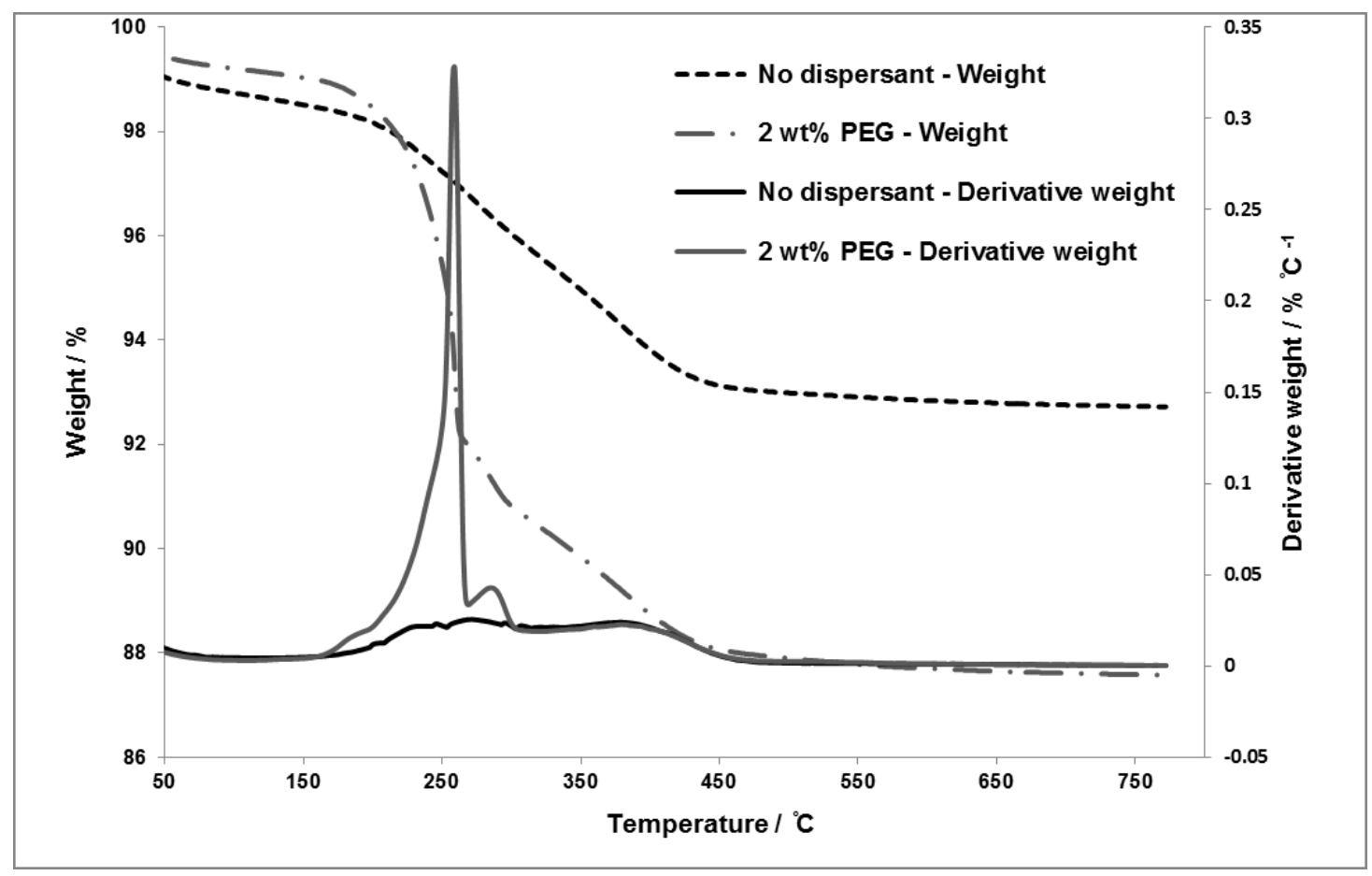

Figure 8: Thermogravimetric analysis of samples with and without added PEG. 


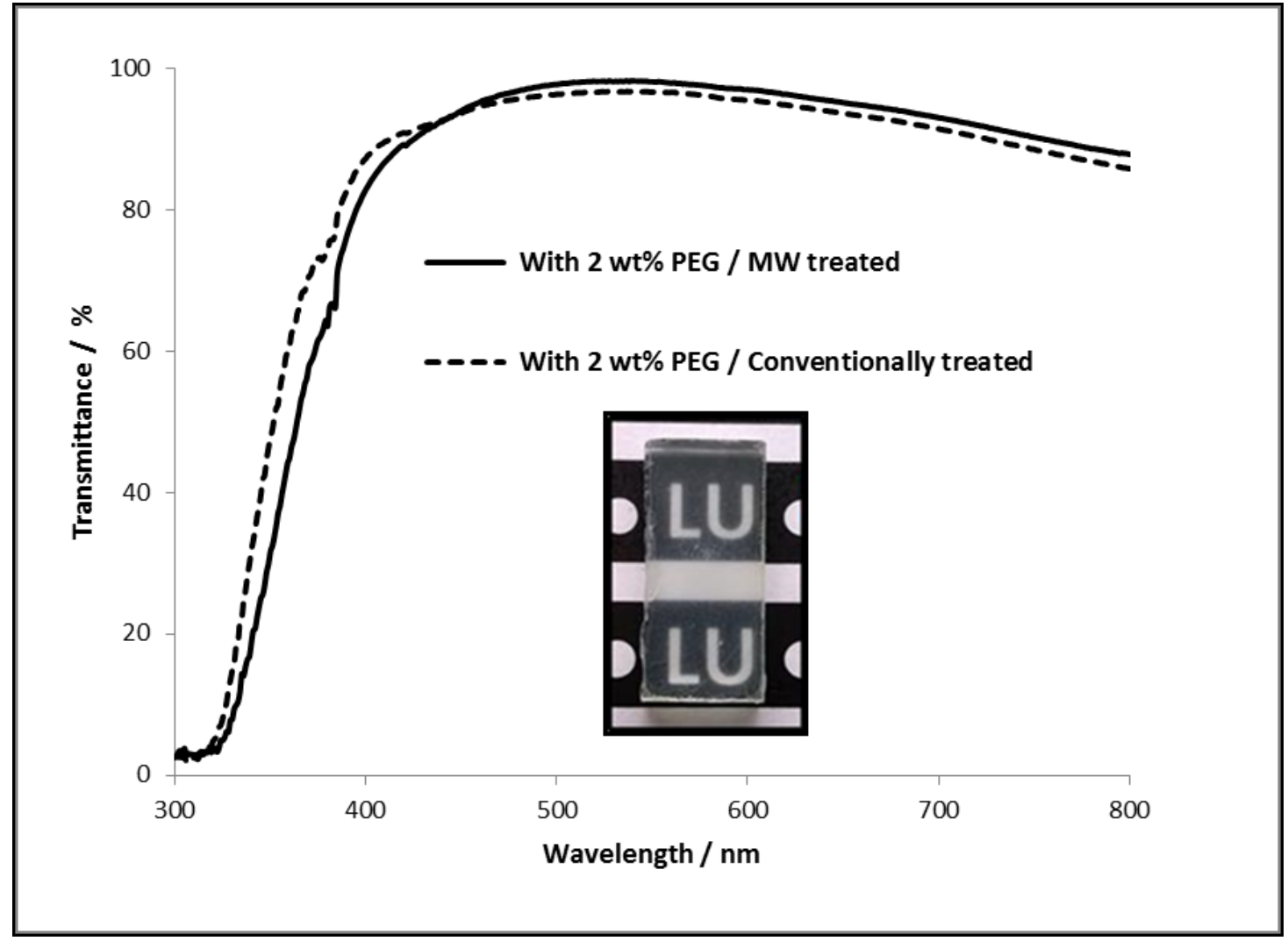

Figure 9: Transmittance spectra of spin coated ITO thin films after conventional and microwave post treatment steps; inset is an image of a glass substrate coated with ITO thin film after post treatment. 